\title{
Contribution of Kinetic Characteristics of Axillary Lymph Nodes to the Diagnosis in Breast Magnetic Resonance Imaging
}

\author{
Şebnem Örgüç1, Işıl Başara', Gökhan Pekindil', Teoman Coşkun² \\ ${ }^{1}$ Department of Radiology, Faculty of Medicine, Celal Bayar University, Manisa, Turkey \\ ${ }^{2}$ Department of General Surgery, Faculty of Medicine, Celal Bayar University, Manisa, Turkey
}

\section{ABSTRACT}

Objective: To assess the contribution of kinetic characteristics in the discrimination of malignant-benign axillary lymph nodes.

Material and Methods: One hundred fifty-five female patients were included in the study. Following magnetic resonance imaging (MRI) examinations postprocessing applications were applied, dynamic curves were obtained from subtracted images. Types of dynamic curves were correlated with histopathological results in malignant cases or final clinical results in patients with no evidence of malignancy. Sensitivity, specificity, positive likehood ratio (+LHR), negative (-LHR) of dynamic curves characterizing the axillary lymph nodes were calculated.

Results: A total of 178 lymph nodes greater than $8 \mathrm{~mm}$ were evaluated in 113 patients. Forty-six lymph nodes in 24 cases had malignant axillary involvement. 132 lymph nodes in 89 patients with benign diagnosis were included in the study. The sensitivity of type 3 curve as an indicator of malignancy was calculated as $89 \%$. However the specificity, +LHR, - LHR were calculated as $14 \%, 1.04,0.76$ respectively.

Conclusion: Since kinetic analysis of both benign and malignant axillary lymph nodes, rapid enhancement and washout (type 3) they cannot be used as a discriminator, unlike breast lesions. MRI, depending on the kinetic features of the axillary lymph nodes, is not high enough to be used in the clinical management of breast cancer patients.

Key Words: Axillary lymph nodes, breast carcinoma, kinetic characteristics, magnetic resonance imaging

\section{Introduction}

Magnetic resonance imaging (MRI) is frequently used in the diagnosis and characterization of suspicious breast lesions. The sensitivity of postcontrast dynamic MRI is high (about 90\%) but the specificity is relatively low (about 37\%$86 \%$ ) in detecting breast carcinoma. Benign lesions showing similar static and dynamic enhancement characteristics to malignant lesions are the main reasons of this low specificity (1). In postcontrast breast MRI, kinetic characteristics together with morphologic characteristics contribute in the discrimination of benign-malignant lesions.

Kinetic curves (time-signal intensity curves) show signal intensity changes of the tissue by time as a reflection of enhancement. Increase of signal and kinetic curves are evaluated in two stages: The early stage is in first 2-3 minutes after bolus injection and the late stage is thereafter. Malignant and benign lesions can be categorized according to these characteristics (2). It is well recognized that most primary breast cancers show rapid enhancement on MRI.

Since axillary lymph node status is the most important prognostic factor in breast cancer patients, characterization of them prior to surgery is crucial. Breast MRI is a potential tool for the evaluation of axillary lymph nodes using the same data. In this study, we aimed to assess the contribution of kinetic characteristics in the discrimination of malignant-benign axillary lymph nodes.

\section{Material and Methods}

Between January 2009 and May 2010, 155 female patients were included in the study. All patients gave informed consent and the study was approved by the Joint Ethical Committee of the two institutions. The ages of the patients ranged between 28-76 years. In malignant diagnosed patients the average of the ages was 45 years, in benign diagnosed patients the average was 43 years.

MRI examinations were carried out in two different imaging centers using the same MRI protocol. Both centers applied conventional breast MRI using 1.5 Tesla MR device Signa HDx; (General Electric, Milwaukee, WI, USA). The routine sequences were axial Short TI Inversion Recovery (STIR), sagittal Fast Spin Echo (FSE) Fat Saturated T2W, sagittal 3D VIBRANT (Postcontrast Fat Saturated T1W). Following MRI examinations postprocessing applications were applied and dynamic curves were obtained from subtracted images. To ensure that the lymph nodes from which the time-intensity curves obtained from 
were the same as the ones dissected in the surgery, the location of each was discussed with the surgeon. Also, the maximum diameters of the lymph nodes were correlated with the pathology specimens. The sensitivity, specificity, positive and negative likelihood ratios of dynamic curves characterizing the axillary lymph nodes were calculated.

\section{Results}

In the study, the ages of the malignant diagnosed and benign diagnosed groups were not statistically different from each other ( $p=0.3$ Student's t test). Lymph nodes were determined in 113 of 155 cases included to the study. Lymph nodes with a diameter of $8 \mathrm{~mm}$ or greater were included in the study. The number of malignant lymph nodes was 46 , benign lymph nodes was 132 . The average diameter was $13.05 \pm 5.48 \mathrm{~mm}$. The average diameter in malignant lymph nodes was $16.82 \pm 8.04 \mathrm{~mm}$. and benign lymph nodes was $11.62 \pm 3.47 \mathrm{~mm}$. In the remaining 42 cases, there was no lymph node measuring more than $8 \mathrm{~mm}$. The malignant axillary lymph nodes were significantly larger than the benign ones ( $p=0.001$ Student's $t$ test).

A total of 178 lymph nodes greater than $8 \mathrm{~mm}$ were evaluated in 113 patients. 46 lymph nodes in 24 cases diagnosed with malignant axillary involvement, and 132 lymph nodes in 89 patients with a benign diagnosis were included in the study. The types of the dynamic curves were correlated with the histopathologic results in malignant cases or final clinical results in patients with no evidence of malignancy. 154 of 178 lymph nodes which were evaluated in our study, had type 3 kinetic curves. In 12 lymph nodes type 2 and in 12 lymph nodes type 1 kinetic curve were detected. The benign lymph nodes showed type 1 kinetic curve in $7.6 \%(n=10)$, type 2 in
$6.8 \%(n=9)$ and type 3 in $85.6 \%(n=113)$. The malignant lymph nodes exhibited type 1 kinetic curve in $4.3 \%(n=2)$, type 2 in $6.5 \%(n=3)$ and type 3 in $89 \%(n=41)$. The distribution of kinetic curve types in malignant and benign axillary lymph nodes is shown in table (Table 1).

Although malignant lesions showed a slightly higher percentage of type 3 kinetic curves, there was no statistically significant difference between the types of kinetic curves in malignant and benign axillary lymph nodes. The prevalence of malignancy in our study group was $25.8 \%$. The sensitivity of type 3 curve as an indicator of malignancy was calculated as $89 \%$. However, the specificity was calculated as $14 \%$. Positive likelihood ratio was 1.04 (95\% confidence level 0.92-1.18), and negative likelihood ratio was 0.76 (95\% confidence level 0.30-1.91).

Representative cases of our study are shown (Figures 1-5).

\section{Discussion}

Kinetic curves (time-signal intensity curves) show signal intensity changes formed in the tissue by time as a reflection of enhancement. Kinetic curves are evaluated for increase of signal in two stages: in the early stage after bolus injection (first 2-3 minutes) and in the late stage (2). In the early stage, the rate of signal increase can be slow, moderate and fast. In the late stage, the intensity can continue to increase (persistent type, type 1- benign type) it can stay at the same level (plateau type, type 2- doubtful type) or it can decrease (wash-out type, type 3-malignant type) (1, 3). In malignant lesions, signal intensity usually increases to the double level of its starting level in $90-120$ seconds $(100 \%)$ or continue to increase and in 3-5 minutes there can be loss of contrast $(1,4)$. The curve types are represented in the figure (Figure 6).

Table 1. The distribution of kinetic curve types in malignant and benign axillary lymph nodes

\begin{tabular}{|lcccc|}
\hline & Type I & Type II & Type III & Number of Cases \\
\hline Benign & $10(7.6 \%)$ & $9(6.8 \%)$ & $113(85.6 \%)$ & 89 \\
Malignant & $2(4.3 \%)$ & $3(6.5 \%)$ & $41(89.1 \%)$ & 24 \\
\hline
\end{tabular}
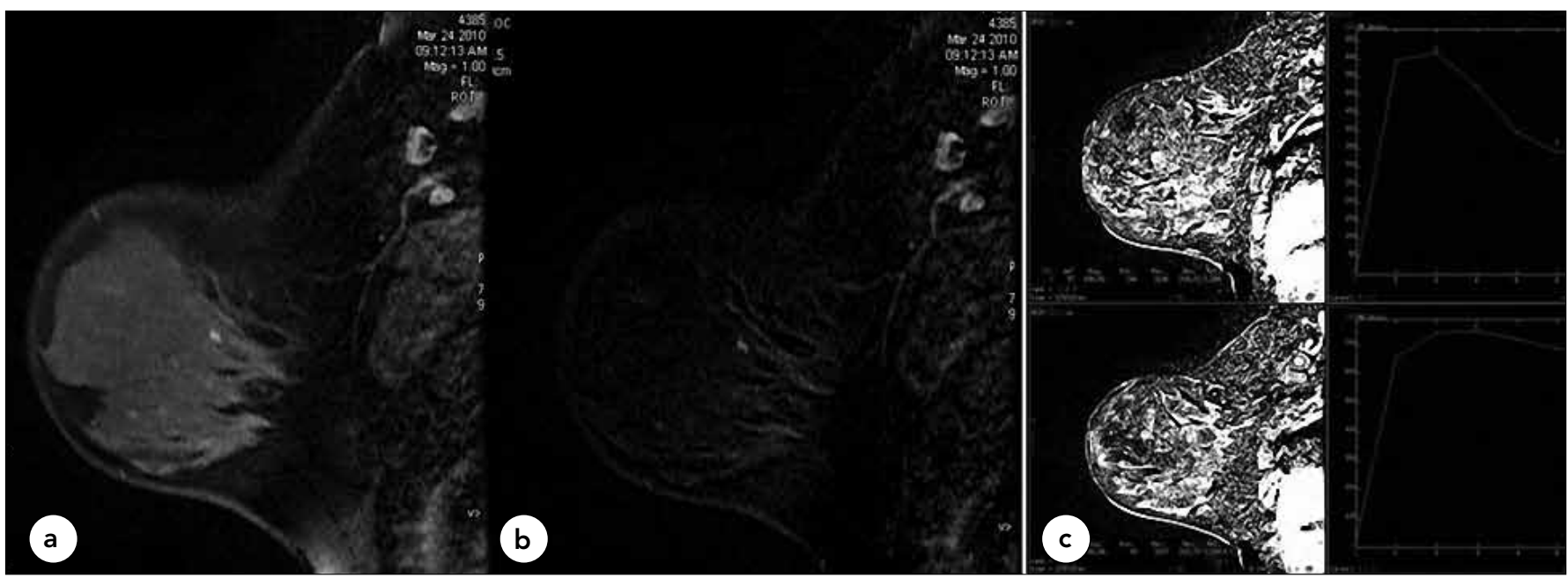

Figure 1. Case 1, Benign, Type 3 Pattern

40-year-old female patient with benign foci of enhancement in breast MRI investigation (BIRADS 2) a. T1W, fat saturated post-contrast sagittal, b. Subtraction Images c. Kinetic curves show multiple reactive axillary lymph nodes with fatty hila and type 3 time intensity curve 


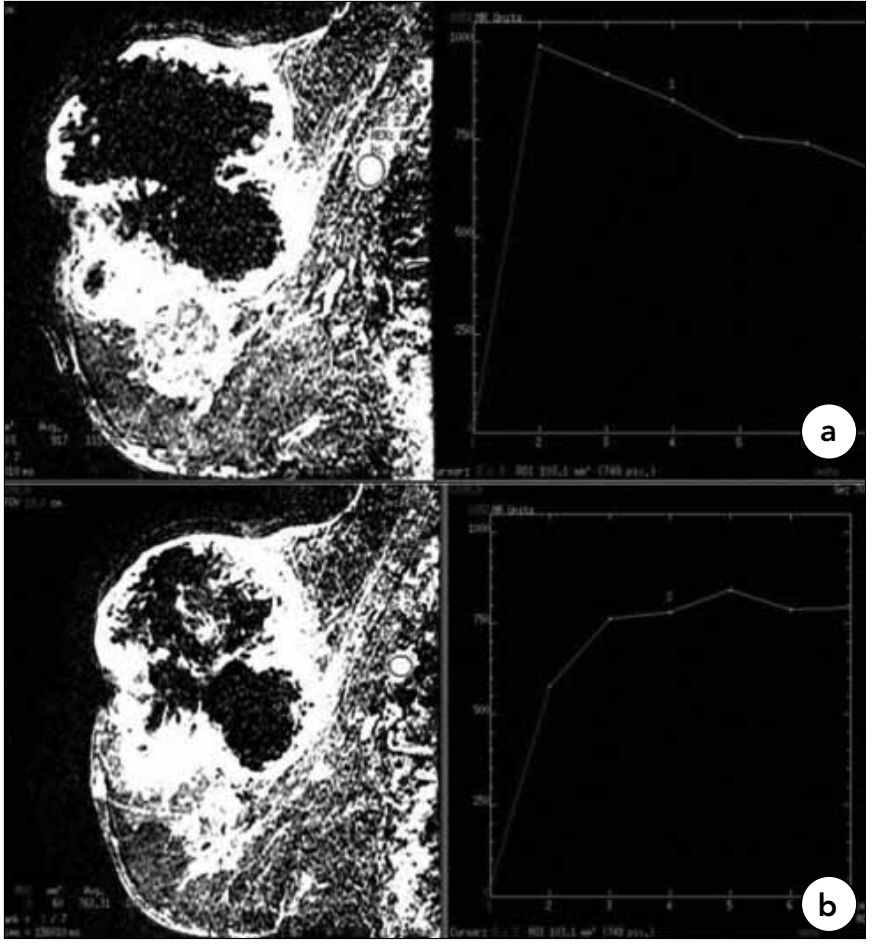

Figure 2. Case 2, Malignant, Type 3 Pattern

a. 37-year-old female patient with invasive ductal carcinoma with giant necrotic mass (BIRADS 5), there is a spherical malignant axillary lymph node. The kinetic curve pattern is type 3 . b. Another malignant lymph node in the same patient with type 2 kinetic curve pattern

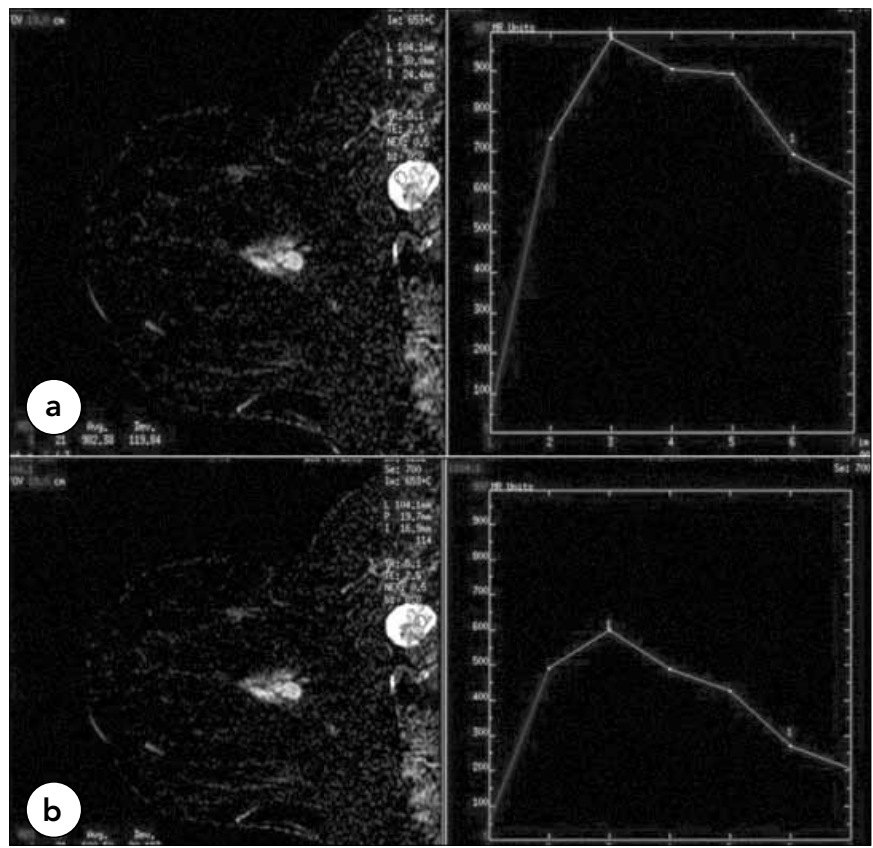

Figure 3. Case 3, Malignant Necrotic Lymph Node, Type 3 a. In the axillary region of a 42-year-old female patient with invasive ductal carcinoma (BIRADS 5), there is a malignant lymph node with central necrosis. The dynamic curve pattern obtained from the wall of the lymph node shows type 3 kinetic curve pattern. b. ROI placed in the more central part of the axillary lymph node displays a type 3 kinetic curve pattern with a lower amplitude

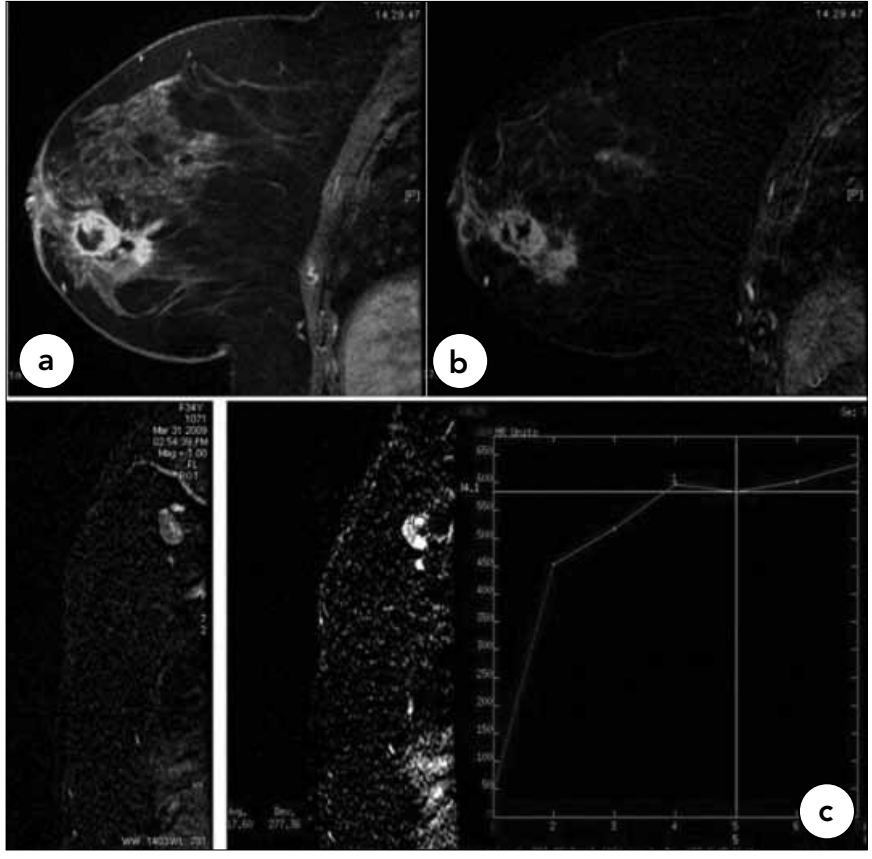

Figure 4. Case 4, Malignant, Type 1

Invasive ductal carcinoma with multifocal necrotic masses in a 54-yearold female patient (BIRADS 5) a. T1W, fat saturated post-contrast, $b$. Subtraction images. c. The malignant axillary lymph nodes show type 1 kinetic curve patterns

The detection of dynamic enhancement curves has increased the specificity of breast MRI markedly. However, malignant and benign lesions may have similar enhancement characteristics. False negative results can be seen in in situ ductal, invasive lobular, invasive ductal with high desmoplastic reaction, some tubular carcinomas and with recent or continuing chemotherapy regimen history cases. These lesions can enhance like a benign type and show low density enhancement. Additionally, no contrast material enhancement can be seen. Some lesions such as fibroadenomas, proliferative fibrocystic changes, fat necrosis, hormonal changes, mastitis, ductal atypia, papilloma, sclerosing adenosis, radial scar, lobulary neoplasia and intramammarian lymph nodes can cause false positive results (4-6).

In dynamic enhanced breast MRI examination, the contribution of kinetic curve patterns to the diagnosis is a known reality. The lesions with type 3 patterns are accepted as malignant and with type 1 patterns are accepted as benign with a high probability $(2,7-10)$.

In most of the lymph nodes evaluated in our study, type 3 pattern kinetic curves were obtained. Type 3 kinetic curve patterns were seen in 113 benign, 41 malignant of a total of 154 lymph nodes. The form of these kinetic curves has a rapid wash-in and a rapid wash-out without a plateau. The sensitivity of the type 3 kinetic curve was calculated as $89 \%$. However, due to the high number of false positive cases, the specificity was calculated as low as $14 \%$.

In the literature there are several studies for the evaluation of axillary lymph nodes on MRI of patients with breast cancer. The studies are mostly about enhancement and MRI characteristics of the lymph nodes (11-15). 


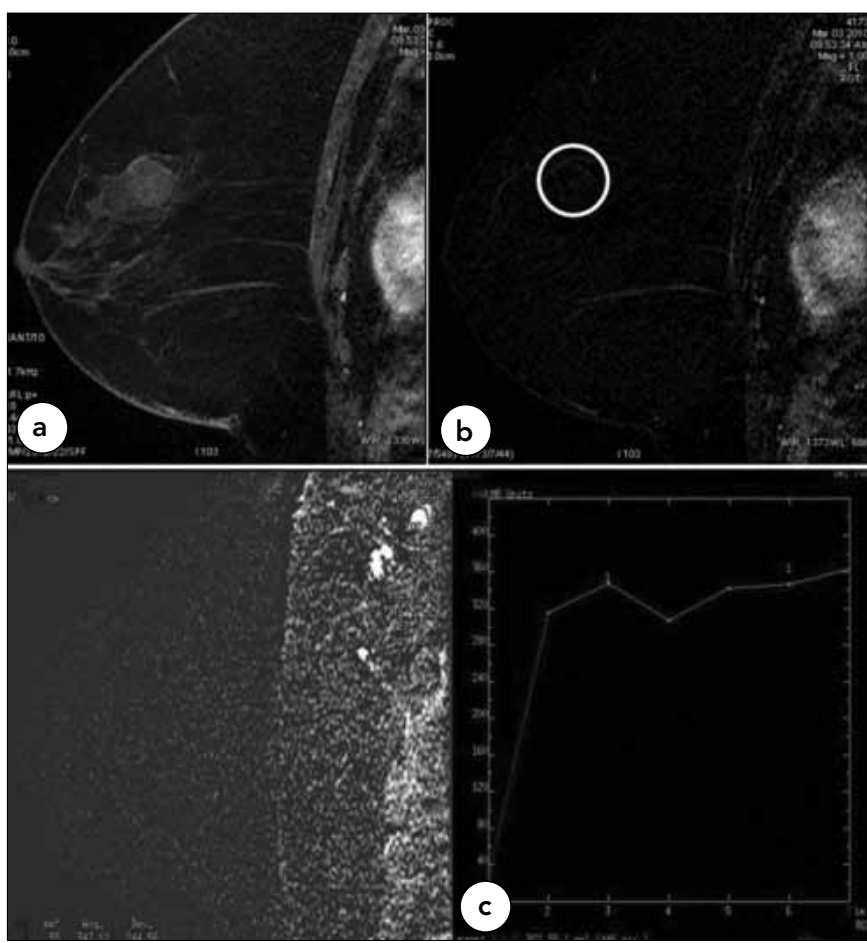

Figure 5. Case 5, Benign, Type 1

Fibroadenoma in a 56-year-old female patient shows no enhancement (BIRADS 2) (in circle), a. T1W, fat saturated post-contrast, b. Subtraction images. c. There are benign axillary lymph nodes with type 1 kinetic curve patterns

In a study by Luciani et al., (11) high-resolution MRI of the axilla and bilateral breast MRI was evaluated in order to identify nodal lymph node features and metastatic involvement.

One of these studies was carried out by Murray et al. (12) They performed a study including 47 female patients with breast carcinoma. In this study, they evaluated MRI and enhancement patterns. They concluded that, in general, patients who are "axillary node positive" have larger nodes that enhance more following contrast material. The usefulness of such an examination depends on the definition of a threshold of significance of some measurement and then calculation of the sensitivity and specificity of the technique.

Memarsadeghi et al. (13) had a study for the assessment of axillary lymph node metastases of patients with breast carcinoma. They evaluated nonenhanced and Ultrasmall Superparamagnetic Iron Oxide (USPIO) enhanced MRI. They included 22 women with breast carcinoma and concluded that USPIO-enhanced MRI appears to be valuable for assessment of axillary lymph node metastases in patients with breast carcinomas and is superior to nonenhanced MRI.

In Bahri et al.'s (14) study, the angiogenetic characteristics of breast masses and relations of these characteristics with axillary metastases were evaluated. Increased angiogenetic tumor activities can be evaluated by MRI. These activities support growth and metastases of cancer. In breast cancer, it may be hypothesized that a tumor with higher angiogenesis may facilitate the spread of cancer cells, and thus is more likely to show lymph node metastasis and earlier recurrence. In Bahri et al.'s (14) study, 62 patients were evaluated. They

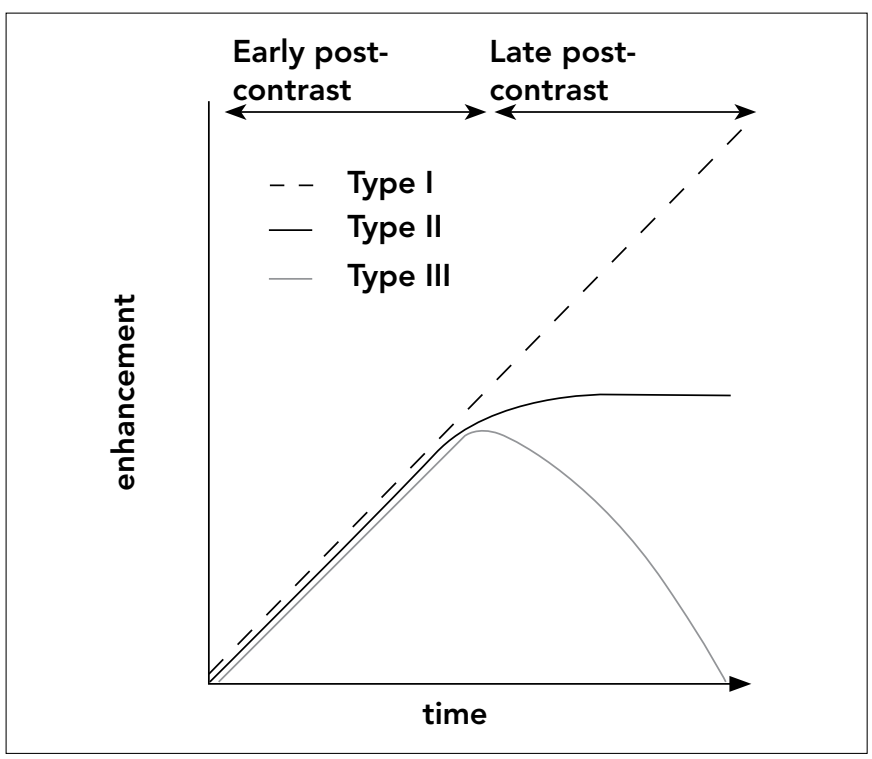

Figure 6. Time-enhancement curves show signal intensity changes formed in the tissue by time as a reflection of enhancement. Kinetic curves are evaluated for increase of signal in two stages: In the early stage after bolus injection (first 2-3 minutes) and in the late stage. In the early stage, the rate of signal increase can be slow, moderate and fast. In the late stage, the intensity can continue to increase (persistent type, type 1-benign type) it can stay at the same level (plateau type, type 2- doubtful type) or it can decrease (wash-out type, type 3-malignant type)

found that the lymph node positive group had a stronger washout than the lymph node negative cohort, suggesting that invasive breast cancers with higher angiogenesis are more likely to have positive nodes. Their results were consistent with those of Tuncbilek et al., (15) which showed a correlation between lymph nodes metastasis and washout slope of the primary tumor.

However, in these studies of axillary lymph nodes, kinetic curve characteristics have not been determined. In our study we evaluated the kinetic characteristics of axillary lymph nodes. Since kinetic analysis of both benign and malignant axillary lymph nodes have rapid enhancement and washout (type 3) they cannot be used as a discriminator, unlike breast lesions. The enhancement kinetics is not a reliable method of predicting absence of axillary nodal metastases in women with breast cancer, thereby avoiding axillary surgery.

\section{Conclusion}

Morphological criteria such as size, shape, loss of fatty hilum, irregular contours indicating extranodal involvement should be kept in the forefront during the evaluation of axillary lymph nodes in breast MRI. When the knowledge of widespread use of sentinel lymph node biopsy and detection of micrometastasis are kept in mind, the accuracy of MRI depending on the kinetic features of the axillary lymph nodes is not high enough to be used in the clinical management of breast cancer patients. 


\section{Conflict of Interest}

No conflict of interest was declared by the authors.

\section{References}

1. Kuhl CK, Mielcareck P, Klaschik S, Leutner C, Wardelmann E, Gieseke J, et al. Dynamic breast MR imaging: are signal intensity time course data useful for differential diagnosis of enhancing lesions? Radiology 1999;211:101-10.

2. Balu-Maestro C. Magnetic resonance imaging of the breast. J Radiol 2001;82:17-26.

3. American College of Radiology. ACR BIRADS - Magnetic Resonance Imaging. In: ACR Breast Imaging Reporting \& Data System, breast imaging atlas. Reston, Va: American College of Radiology, 2003.

4. De Bruhl ND, Michael D, Basset LW. Magnetic resonance imaging of breast tumors. In: Basset LW, Jackson VP, Fu KL, Fu YS, eds. Diagnosis of Diseases of the Breast 2nd ed. Elsevier Saunders: Philadelphia, 2005; 225-50.

5. Morris EA. Breast cancer imaging with MRI. Radiol Clin North Am 2002;40:443-66. [CrossRef]

6. Daniel BL, Ikeda DM. Magnetic resonance imaging of breast cancer and MRI-guided biopsy. In: Ikeda DM ed. The requisities: Breast Imaging. Elsevier-Mosby: Philadelphia 2004;189-224.

7. Boetes C, Barentsz JO, Mus RD, van der Sluis, van Erning LJ, Hendriks $\mathrm{JH}$, et al. MR characterization of suspicious breast lesions with a gadolinium- enhanced TurboFLASH subtraction technique. Radiology 1994;193:777-81.
8. Bartella L, Liberman L, Morris EA, Dershaw DD. Nonpalpable mammographically occult invasive breast cancers detected by MRI. AJR Am J Roentgenol 2006;186:865-70. [CrossRef]

9. Schnall MD, Blume J, Bluemke DA, DeAngelis GA, DeBruhl N, Harms $S$, et al. Diagnostic architectural and dynamic features at breast MR imaging: multicenter study. Radiology 2006;238: 42-53. [CrossRef]

10. Negendank W. Studies of human tumors by MRS: a review. NMR Biomed 1992;5:303-24. [CrossRef]

11. Luciani A, Dao TH, Lapeyre M, Schwarzinger M, Debaecque $C$, Lantieri $L$, et al. Simultaneous bilateral breast and high-resolution axillary MRI of patients with breast cancer: preliminary results. AJR Am J Roentgenol 2004;182:1059-67.

12. Murray AD, Staff T, Redpath TW, Gilbert FJ, Ah-See AK, Brookes $J A$, et al. Dynamic contrast enhanced MRI of the axilla in women with breast cancer: comparison with pathology of excised nodes. Br J Radiol 2002;75:220-8.

13. Memarsadeghi M, Riedl CC, Kaneider A, Galid A, Rudas M, Matzek W, et al. Axillary lymph node metastases in patients with breast carcinomas: assessment with nonenhanced versus uspioenhanced MR imaging. Radiology 2006;241:367-77. [CrossRef]

14. Bahri S, Chen JH, Yu HJ, Kuzucan A, Nalcioglu O, Su MY. Can dynamic contrast-enhanced MRI (DCE-MRI) predict tumor recurrence and lymph node status in patients with breast cancer? Ann Oncol 2008; 19:822-4. [CrossRef]

15. Tuncbilek N, Karakas HM, Okten OO. Dynamic magnetic resonance imaging in determining histopathological prognostic factors of invasive breast cancers. Eur J Radiol 2005;53:199-205. [CrossRef] 